\title{
Basidiobolus omanensis sp. nov. Causing Angioinvasive Abdominal Basidiobolomycosis
}

\author{
Abdullah M. S. Al-Hatmi 1,2,3,*(D), Abdullah Balkhair ${ }^{4}$, Ibrahim Al-Busaidi ${ }^{4} \mathbb{D}$, Marcelo Sandoval-Denis ${ }^{5}$, \\ Saif Al-Housni ${ }^{1}$, Hashim Ba Taher ${ }^{4}$, Asmaa Hamdan Al Shehhi ${ }^{6}$, Sameer Raniga ${ }^{7}$ (D, Maha Al Shaibi ${ }^{8}$, \\ Turkiya Al Siyabi ${ }^{9}$, Jacques F. Meis ${ }^{3,10}{ }^{D}$, G. Sybren de Hoog ${ }^{3} \mathbb{D}$, Ahmed Al-Rawahi ${ }^{1}$, Zakariya Al Muharrmi ${ }^{9}$, \\ Ahmed Al-Harrasi ${ }^{1}$ (D) and Badriya Al Adawi ${ }^{9, *}$
}

check for updates

Citation: Al-Hatmi, A.M.S.; Balkhair, A.; Al-Busaidi, I.; Sandoval-Denis, M.; Al-Housni, S.; Ba Taher, H.; Al Shehhi, A.H.; Raniga, S.; Al Shaibi, M.; $\mathrm{Al}$ Siyabi, T.; et al. Basidiobolus omanensis sp. nov. Causing Angioinvasive Abdominal Basidiobolomycosis. J. Fungi 2021, 7, 653. https:// doi.org/10.3390/jof7080653

Academic Editor: David S. Perlin

Received: 3 July 2021

Accepted: 9 August 2021

Published: 12 August 2021

Publisher's Note: MDPI stays neutra with regard to jurisdictional claims in published maps and institutional affiliations.

Copyright: (c) 2021 by the authors. Licensee MDPI, Basel, Switzerland. This article is an open access article distributed under the terms and conditions of the Creative Commons Attribution (CC BY) license (https:// creativecommons.org/licenses/by/ $4.0 /$ )
1 Natural \& Medical Sciences Research Center, University of Nizwa, Nizwa 616, Oman; saifalhousni@unizwa.edu.om (S.A.-H.); ahmed@unizwa.edu.om (A.A.-R.); aharrasi@unizwa.edu.om (A.A.-H.)

2 Department of Biological Sciences \& Chemistry, College of Arts and Sciences, University of Nizwa, Nizwa 616, Oman

3 Centre of Expertise in Mycology, Radboud University Medical Centre/Canisius Wilhelmina Hospital, 6532 SZ Nijmegen, The Netherlands; jacques.meis@gmail.com (J.F.M.); sybren.dehoog@radboudumc.nl (G.S.d.H.)

4 Infectious Diseases Unit, Department of Medicine, Sultan Qaboos University Hospital, Muscat 123, Oman; balkhair2020@gmail.com (A.B.); ibrahimbusaidi@gmail.com (I.A.-B.); Bataher84@gmail.com (H.B.T.)

5 Westerdijk Fungal Biodiversity Institute, 3584 CT Utrecht, The Netherlands; m.sandoval@wi.knaw.nl

6 Department of Pathology, Sultan Qaboos University Hospital, Muscat 123, Oman; Asmaah@squ.edu.om

7 Department of Radiology and Molecular Imaging, Sultan Qaboos University Hospital, Muscat 123, Oman; sameerraniga@yahoo.com

8 Department of Surgery, Sultan Qaboos University Hospital, Muscat 123, Oman; maha.alshaibi@squ.edu.om

9 Department of Microbiology and Immunology, Sultan Qaboos University Hospital, Muscat 123, Oman; dr.alsiyabi@gmail.com (T.A.S.); almuharrmi@gmail.com (Z.A.M.)

10 Departments of Medical Microbiology and Infectious Diseases, Canisius Wilhelmina Hospital, 6532 SZ Nijmegen, The Netherlands

* Correspondence: a.alhatmi@unizwa.edu.om (A.M.S.A.-H.); badriyak@squ.edu.om (B.A.A.); Tel.: +968-24144997 (B.A.A.); Fax: +968-24144885 (B.A.A.)

Abstract: Human infectious fungal diseases are increasing, despite improved hygienic conditions. We present a case of gastrointestinal basidiobolomycosis (GIB) in a 20-year-old male with a history of progressively worsening abdominal pain. The causative agent was identified as a novel Basidiobolus species. Validation of its novelty was established by analysis of the partial ribosomal operon of two isolates from different organs. Phylogeny of ITS and LSU rRNA showed that these isolates belonged to the genus Basidiobolus, positioned closely to B. heterosporus and B. minor. Morphological and physiological data supported the identity of the species, which was named Basidiobolus omanensis, with CBS 146281 as the holotype. The strains showed high minimum inhibitory concentrations (MICs) to fluconazole ( $>64 \mu \mathrm{g} / \mathrm{mL})$, itraconazole and voriconazole $(>16 \mu \mathrm{g} / \mathrm{mL})$, anidulafungin and micafungin $(>16 \mu \mathrm{g} / \mathrm{mL})$, but had a low MIC to amphotericin B $(1 \mu \mathrm{g} / \mathrm{mL})$. The pathogenic role of B. omanensis in gastrointestinal disease is discussed. We highlight the crucial role of molecular identification of these rarely encountered opportunistic fungi.

Keywords: gastrointestinal; basidiobolomycosis; phylogeny; ITS; LSU; morphology; Basidiobolus

\section{Introduction}

Basidiobolomycosis is a rare infection in humans that usually affects skin and subcutaneous tissues of limbs and trunk. The disorder prevalently occurs in immunocompetent individuals. The first case of subcutaneous basidiobolomycosis caused by Basidiobolus ranarum was reported from Indonesia in 1956 [1]. Prior to this report, van Overeem [2] described a case in a horse by an as yet unidentified Basidiobolus species. Infections have 
also been reported in dogs [3]. Human gastrointestinal basidiobolomycosis (GIB) is increasingly recognised in immunocompromised or otherwise debilitated patients [4-7]. This deep infection, recently described from the Middle East [7], may take a serious course. The authors analysed 28S rDNA sequences of five strains and found them to cluster in a monophyletic branch close to B. haptosporus, having $99.97 \%$ similarity with the latter species and slightly lower $(99.25 \%)$ with B. ranarum. Given the low variability of the used marker, these results suggest a possibly undescribed Basidiobolus species.

Basidiobolus has classically been listed as a member of the phylum Zygomycota. On the basis of the molecular phylogeny of small subunit ribosomal RNA, a major taxonomic change has been proposed [8]. Arbuscular mycorrhizal fungi were removed from Zygomycota and placed into a separate monophyletic phylum, the Glomeromycota. This triggered a further subdivision of Zygomycota as part of a comprehensive phylogenetic classification of the kingdom Fungi [9]. Acknowledging the high diversity of the phylum, taxa conventionally attributed to Zygomycota were distributed among separate high-level taxa. As no overarching phylum could be determined, the groups were classified as four subphyla, i.e., Entomophthoromycotina, Mucoromycotina, Kickxellomycotina and Zoopagomycotina [10]. Using traditional taxonomic characteristics in addition to phylogeny, Humber [11] raised the Entomophthoromycotina to the phylum level as Entomophthoromycota, containing three classes (Basidiobolomycetes, Neozygitomycetes and Entomophthoromycetes). Gryganskyi et al. [12] confirmed that Entomophthoromycota is a monophyletic group and is not closely related to any of the flagellate aquatic fungi (i.e., Chytridiomycota or Blastocladiomycota). The study was later elaborated with five genes (LSU, SSU, RPB2, mtSSU, ITS), enabling positioning of the entomophthoralean fungi as a monophyletic group basal to the Mucorales [13]. Five generic lineages were recognised, Batkoa, Basidiobolus, Conidiobolus, Entomophthora and Zoophthora. Spatafora et al. [14] analysed genome data with 192 proteins for 46 taxa including 25 zygomycetes and proposed two further phyla, Mucormycota and Zoopagomycota. This upgrading of the classical groups of Zygomycota during the last two decades underlines the high phenotypic and phylogenetic diversity of these fungi, despite the relatively low number of extant species when compared with, e.g., the Ascomycota.

Basidiobolus and Conidiobolus are sister genera but at large phylogenetic distance, both containing members that are able to cause human infections [15]. Basidiobolus was introduced in the late 19th century [16] and contains ubiquitous species residing in (sub)tropical marshes. They are commonly isolated from intestines and the dung of amphibians and reptiles [17], and also occur in the intestines of warm-blooded vertebrates, including humans $[15,18]$. Based on LSU, SSU, RPB2, mtSSU and ITS sequences, six species are currently distinguished: $B$. haptosporus, B. heterosporus, B. magnus, B. meristosporus, B. microsporus and $B$. ranarum [17]. However, studies of antigens and restriction enzyme profiles suggested that all human-pathogenic isolates belong to a single species, B. ranarum $[19,20]$. The taxonomy of Basidiobolus is a much-debated issue. Unlike many other filamentous fungi, only few phenotypic characteristics are available to differentiate Basidiobolus species. The genus was previously classified in the order Entomophthorales, but was recently assigned to the order Basidiobolales because of its unresolved position close to the flagellated genus Olpidium $[9,21]$.

The recent report of gastrointestinal basidiobolomycosis from Saudi Arabia [7] was carefully analysed using $28 \mathrm{~S}$ rDNA gene sequences of five strains. These were found to cluster in a monophyletic branch close to B. haptosporus, having $99.97 \%$ similarity with the latter species and slightly lower $(99.25 \%)$ with $B$. ranarum. Given the low variability of the used marker, these results suggest that possibly an undescribed Basidiobolus species was concerned. Here, we report the isolation of a similar Basidiobolus species from a human infection in Oman obtained with two strains from different anatomical sites of a single GIB patient. We introduce it as a novel Basidiobolus species, characterised by morphological features and a deviating multilocus sequence profile. 


\section{Materials and Methods}

\subsection{Phenotypic Studies}

Fungal strains were isolated from a thrombus that was extracted from the common femoral artery, and from urine. Both strains were sent to the Centraalbureau voor Schimmelcultures (CBS) reference collection housed at the Westerdijk Fungal Biodiversity Institute, Utrecht, The Netherlands and deposited under the accession numbers CBS 146281 and CBS 146282, respectively. The strains were cultured on malt extract agar (MEA; Oxoid, Basingstoke, UK), potato dextrose agar (PDA; Oxoid) and Sabouraud glucose agar (SGA; Merck, Darmstadt, Germany). Culture plates were incubated at $25{ }^{\circ} \mathrm{C}$ and $30{ }^{\circ} \mathrm{C}$ for $2-5$ days. Slides were examined with a Nikon Eclipse 80i light microscope, and pictures were taken using a camera attached to the microscope (Nikon; digital-sight DS-5 M, (Nikon Corporation, Tokyo, Japan).

\subsection{DNA Amplification and Sequencing}

For molecular identification, DNA was extracted from a fresh culture grown on MEA plates using the cetyltrimethyl ammonium bromide (CTAB) protocol of Möller et al. [22]. The rDNA internal transcribed spacer region (ITS) and the large ribosomal subunit (LSU) were amplified using the primer pairs ITS1/ITS4 and LR0R/LR5 [23-25]. Sequencing was carried out in both strand directions using the same primer pairs on an Applied Biosystems, Hitachi 3730xl DNA analyser (Applied Biosystems, Foster City, CA, USA). Consensus sequences were assembled using Seqman Pro v. 10.0.1 (DNASTAR, Madison, WI, USA). All newly generated sequences were submitted to GenBank (Table 1).

\subsection{Phylogenetic Inference}

Sequence alignments for ITS and LSU regions were generated using MAFFT v7 [26] and analysed using Maximum Likelihood (ML) and Bayesian (BI) algorithms in the CIPRES Science Gateway portal (www.phylo.org, accessed on 18 December 2020) [27]. Maximum Likelihood analyses were run using RAxML v8.2.10 (randomised accelerated maximum likelihood for high performance computing) [28] with default parameters, and node support values were estimated using rapid bootstrapping (BS) with the number of iterations set automatically by the software. Bayesian analyses were run using MrBayes v3.2.6, [29,30] and consisted of four parallel runs of 5 million generations starting from a random tree topology, sampling trees every 1000 generations. Evolutionary models for each dataset were estimated using MrModeltest v2.3 [31]. The 50\% majority rule consensus trees and posterior probability (PP) values were calculated after discarding the initial $25 \%$ of saved trees as the 'burn-in' fraction. Individual gene phylogenies were compared to search for conflicts between significantly supported clades (ML-BS $\geq 70 \%$, BI-PP $\geq 0.95$ ), after which the two datasets were concatenated and analysed $[32,33]$.

\subsection{Antifungal Susceptibility}

Antifungal susceptibility testing (AFST) of CBS 146281 and CBS 146282 was performed using broth microdilution as described in the CLSI document M38-A2. [34]. The following drugs were used; amphotericin B (Sigma-Aldrich, St. Louis, MO, USA), fluconazole (Pfizer, Groton, CT, USA), itraconazole (Janssen Pharmaceutica, Tilburg, The Netherlands), voriconazole (Pfizer), micafungin (Astellas, Ibaraki, Japan) and anidulafungin (Pfizer). Final concentrations of antifungal agents in the wells ranged from 0.016 to $16 \mu \mathrm{g} / \mathrm{mL}$ for amphotericin B, voriconazole, itraconazole, from 0.016 to $64 \mu \mathrm{g} / \mathrm{mL}$ for fluconazole and 0.008 to $8 \mu \mathrm{g} / \mathrm{mL}$ for anidulafungin and micafungin. Stock solutions of drugs were prepared in dimethyl sulfoxide, except for caspofungin and micafungin, which were dissolved in sterile water and stored at $-80^{\circ} \mathrm{C}$ until use. The strains were grown on potato dextrose agar (PDA, Difco) and incubated at $35^{\circ} \mathrm{C}$ for 5 to 7 days for adequate sporulation. Three reference strains (Paecilomyces variotii ATCC 22319, Candida krusei ATCC 6258, and Candida parapsilosis ATCC 22019) were included as quality controls. 
Table 1. Strain information and accession numbers of DNA sequences included in the phylogenetic analyses.

\begin{tabular}{|c|c|c|c|c|c|}
\hline \multirow{2}{*}{ Species Epithet } & \multirow{2}{*}{ Strain ${ }^{1}$} & \multirow{2}{*}{ Host/Substrate } & \multirow{2}{*}{ Country } & \multicolumn{2}{|c|}{ Sequence Accession Number ${ }^{2}$} \\
\hline & & & & ITS & LSU \\
\hline \multirow{3}{*}{ B. haptosporus } & $\begin{array}{c}\text { ARSEF } 261 \text { = NRRL } 1232 \\
=\text { RSA } 228\end{array}$ & Human & Indonesia & EF392520 & MH869969 \\
\hline & ARSEF 6380 & Soil and litter & UK & EF392528 & MH869969 \\
\hline & ARSEF 8309 & Unknown & USA & EF392531 & EF392420 \\
\hline \multirow[b]{2}{*}{ B. heterosporus } & ARSEF 262 = RSA 964 & Oak leaf & USA & EF392521 & EF392411 \\
\hline & $\begin{array}{c}\text { CBS } 311.66=\text { ATCC } 16580 \\
=\text { IMI } 118288=\text { NRRL } 3687 \\
(\text { ex-type })\end{array}$ & Plant debris & India & MH858801 & JX242587 \\
\hline B. magnus & $\begin{array}{c}\text { CBS } 205.64=\text { ATCC } 15379 \\
=\text { NRRL } 3734 \text { (ex-type) }\end{array}$ & Plant debris & USA & NR_077175 & EF392423 \\
\hline \multirow[b]{2}{*}{ B. meristosporus } & CBS 931.73 & Gecko dung & Ivory Coast & MT830914 & MT831974 \\
\hline & CBS 930.73 & $\begin{array}{c}\text { Ant cemetery of } \\
\text { Paltothyreus tarsatus }\end{array}$ & Ivory Coast & MT830915 & MT831975 \\
\hline \multirow[b]{2}{*}{ B. microsporus } & ARSEF 265 = RSA 962 & Lizard dung & USA & EF392523 & DQ364202 \\
\hline & $\begin{array}{c}\text { CBS } 130.62=\text { ATCC } 14708 \\
=\text { DSM } 3120=\text { IMI } 093345 \\
=\text { RSA } 977 \text { (ex-type) }\end{array}$ & Lizard dung & USA & NR_159603 & MH869698 \\
\hline B. minor & $\begin{array}{l}\text { CBS } 310.66=\text { ATCC } 16579 \\
=\text { IMI } 118287=\text { NRRL } 3677 \\
=\text { NBRC } 109014 \text { (ex-type) }\end{array}$ & Ficus fruit & India & EF392535 & EF392424 \\
\hline \multirow[t]{2}{*}{ B. omanensis } & CBS 146281 (ex-type) & $\begin{array}{c}\text { human intravascular } \\
\text { thrombus }\end{array}$ & Oman & MT830913 & MT831973 \\
\hline & CBS 146282 & Human urine & Oman & MT830912 & MT831972 \\
\hline \multirow{3}{*}{ B. ranarum } & AFTOL-ID 301 & Unknown & Unknown & AY997030 & DQ273807 \\
\hline & CBS 538.63 & Frog & Canada & MH858348 & MH869969 \\
\hline & $\begin{array}{c}\text { CBS } 117.29=\text { ATCC } 11230 \\
=\text { IFO } 9117\end{array}$ & Unknown & Unknown & IF00911701 ${ }^{\mathrm{a}}$ & IF00911701 ${ }^{a}$ \\
\hline Conidiobolus sp. & ARSEF 7942 & Leaf litter & USA & EF392537 & KC788411 \\
\hline
\end{tabular}

${ }^{1}$ AFTOL-ID = Assembling the Fungal Tree of Life, Department of Biology, Duke University, NC, USA; ARSEF = ARS Collection of Entomopathogenic Fungi, USDA-ARS BioIPM Research Unit, Ithaca, NY, USA; ATCC = American Type Culture Collection, Manassas, VA, USA; CBS = Westerdijk Fungal Biodiversity Institute, Utrecht, The Netherlands; DSM = German Collection of Microorganisms and Cell Cultures $\mathrm{GmbH}$, Braunschweig, Germany; IFO = Institute for Fermentation, Osaka, Japan; IMI = CABI Bioscience, Egham, Surrey, UK; NBRC = NITE Biological Resource Center, Chiba, Japan; NRRL = ARS Culture Collection, USDA-ARS National Center for Agricultural Utilization Research, Peoria, IL, USA; RSA = Rancho Santa Ana Botanic Garden, Claremont, CA, USA. ${ }^{2}$ Unless otherwise specified, accession numbers refer to GenBank. ${ }^{a}$ Accession numbers from NBRC sequence database. Newly generated sequences are highlighted in bold.

\section{Case Report and Results}

\subsection{Case Presentation}

A 20-year-old Omani male with type I diabetes mellitus presented to a local hospital with a two-week history of progressively worsening abdominal pain. A computed tomography (CT) scan of the abdomen revealed a complex cecal mass adherent to the pelvic floor and to the terminal ileum with multiple hepatic lesions. The patient underwent an exploratory laparotomy which revealed cecal perforation, an inflammatory mass at the base of the mesentery of the transverse colon, and extensive cecal, terminal ileum and infrahepatic inflammatory processes. Diverting loop ileostomy and drainage of the pelvic collections were performed and biopsies from multiple sites were taken for histopathological examination and for cultures. Initial histopathological examination of the intraoperative samples demonstrated fungal elements with necrosis and angioinvasion, consistent with mucormycosis. The patient was treated with liposomal amphotericin $7.5 \mathrm{mg} / \mathrm{kg} /$ day in 
combination with caspofungin $50 \mathrm{mg} /$ day. Two days postoperatively, the patient suffered acute ischemia of the right lower limb resulting from an extensive thrombus with total occlusion of the right external iliac artery. He was transferred to Sultan Qaboos University Hospital (SQUH) for further care.

Upon presentation at SQUH, physical examination showed a conscious, ill-looking patient with pallor and signs of dehydration. He was afebrile but tachycardic (pulse rate: $104 / \mathrm{min}$ ) and tachypnoeic (respiratory rate: $22 / \mathrm{min}$ ) with oxygen saturation of $95 \%$ in ambient air. Blood pressure was 117/66 mm Hg. Examination of the abdomen revealed a mildly distended abdomen with midline laparotomy scar, and a functioning ileostomy with a healthy stoma. On palpation, there was right-sided abdominal tenderness with no peritoneal irritation signs. Examination of the lower limbs demonstrated signs of acute ischemia of the right leg with absent femoral, popliteal, pedal and posterior tibial pulses. Other systemic examination was within normal limits. Initial laboratory investigations showed a haemoglobin of $8.9 \mathrm{~g} / \mathrm{dL}$, and a total white cell count of 16,700 cells $/ \mu \mathrm{L}$ with an eosinophil count of 1000 cells $/ \mu \mathrm{L}$. Biochemistry panel was normal with the exception of alkaline phosphatase of 372 unit/L. Glycosylated haemoglobin was 5.5\% (within normal range). HIV serology was negative.

The patient underwent urgent right common femoral artery thrombectomy and embolectomy. Multiplanar CT scan of abdomen and pelvis were performed with oral and intravenous contrast (Figure 1).

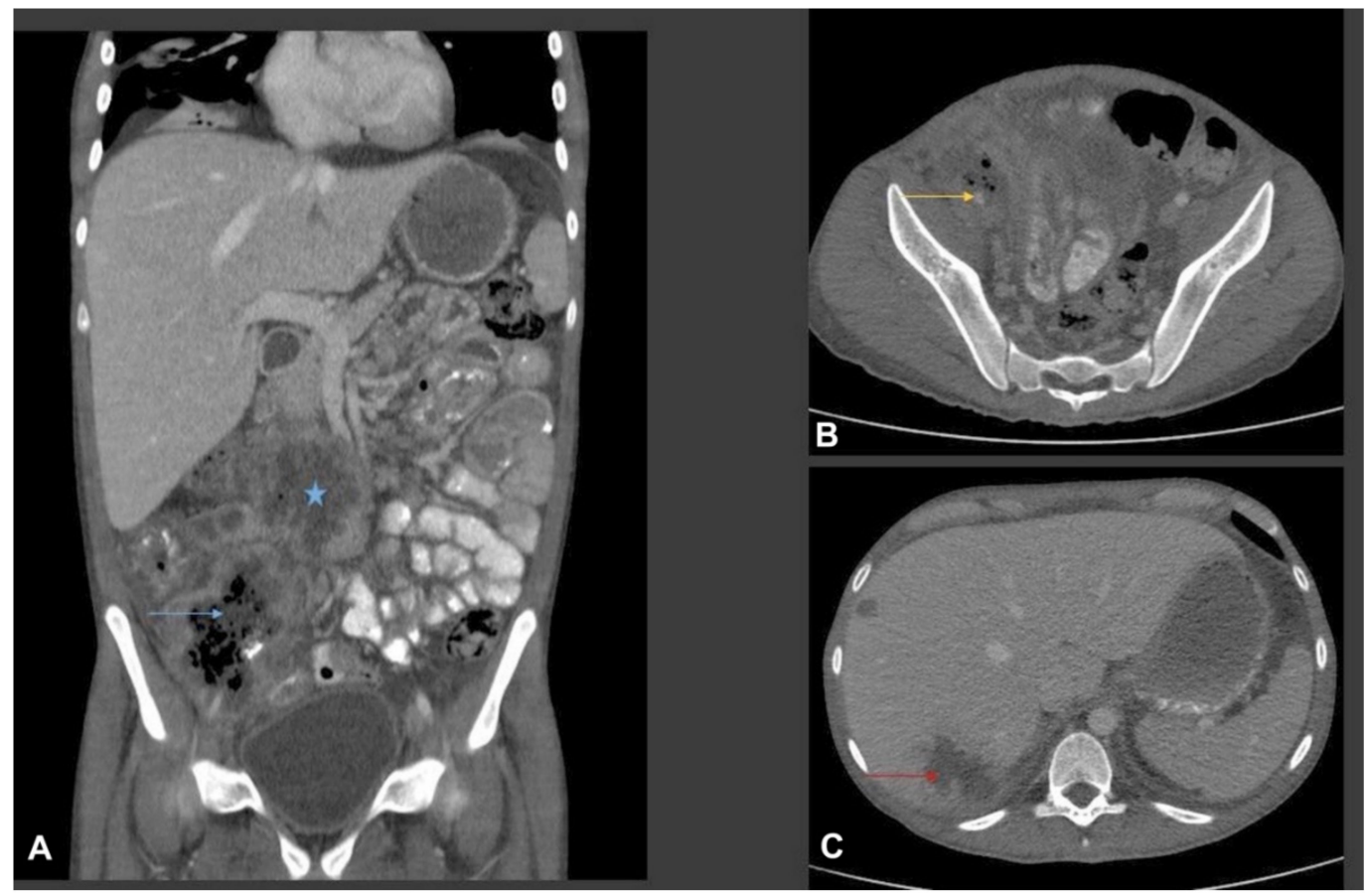

Figure 1. Multiplanar CT scan of abdomen with oral and intravenous contrast. (A) Coronal CT image shows a multispatial ill-defined low-attenuation ring enhancing collection in the root of the mesentery (star) and in the right iliac fossa. The right iliac fossa collection shows thick irregular rim enhancement with central bubbly air lucency (blue arrow). Bowel loops are adherent to the collections. There is marked inflammatory fat stranding noted in the right iliac fossa and right side of the pelvic cavity. Small volume ascites. (B) Axial CT image from the right iliac fossa and pelvis shows marked phlegmonous soft tissue lesion with fat stranding. The phlegmonous inflammatory mass is encasing the right external iliac artery which is small and irregular in calibre (yellow arrow). (C) Axial images from the liver show multifocal ill-defined low-attenuation liver lesions of variable size (two of them shown in this image). The largest was seen in the subcapsular liver in the segment VII and shows surround oedema, suggestive of liver abscess (red arrow).

Then, a limited exploratory laparotomy was performed and entailed right hemicolectomy, drainage of infrahepatic collections and an abdominal wash. Samples from both 
surgical procedures were sent for histopathology examination and culture. High-dose liposomal amphotericin $(7.5 \mathrm{mg} / \mathrm{kg} /$ day $)$ in combination with caspofungin $50 \mathrm{mg} /$ day were continued. When empirically treating cases of life-threatening mucormycosis, we use this antifungal combination based on some evidence suggesting synergistic action.

Culture of the thrombus that was extracted from the common femoral artery grew a mould that was identified phenotypically as Basidiobolus species (Figure 2).

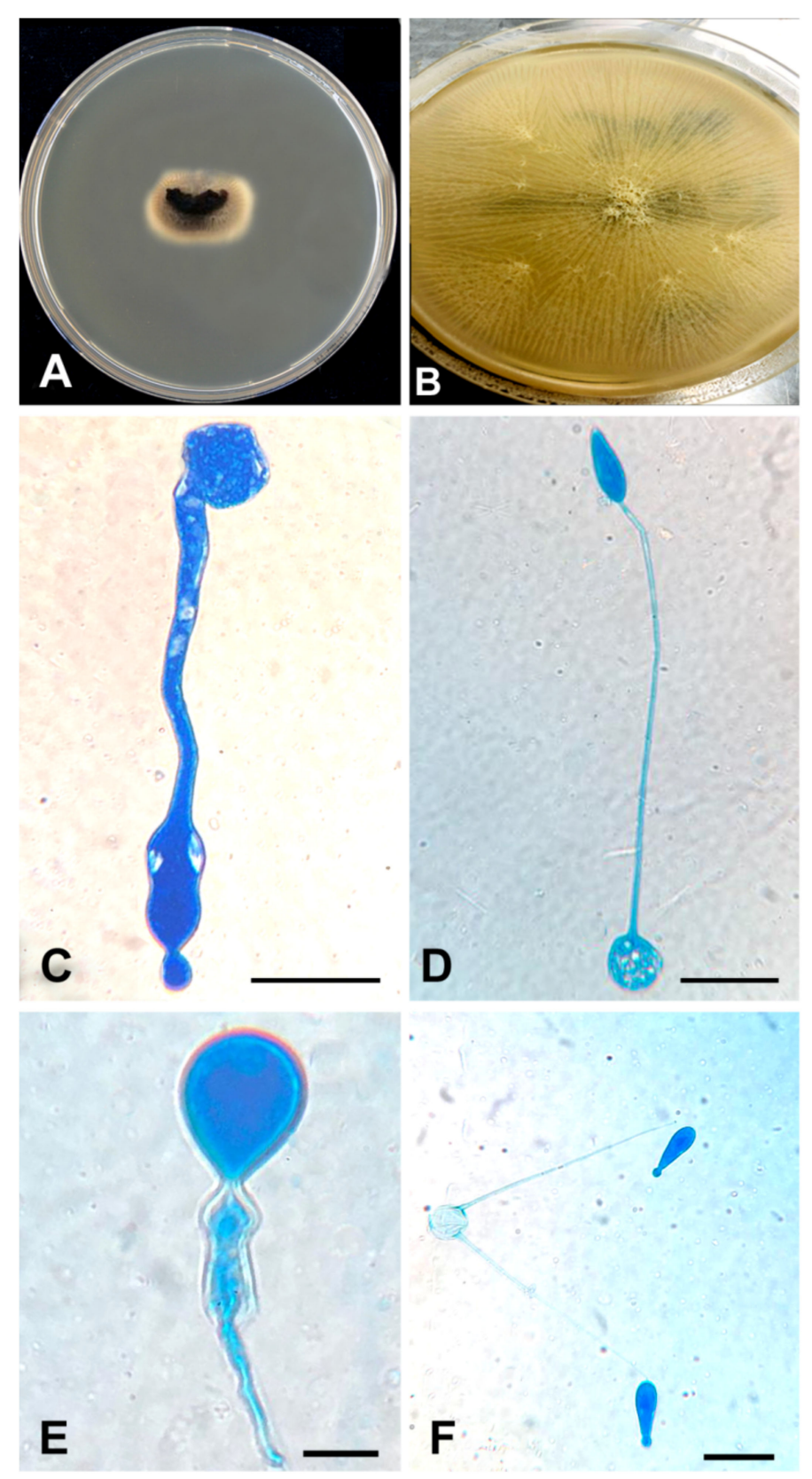

Figure 2. (A) Direct culture (SAB) from the thrombus. A piece of the thrombus (the black material at the centre) was put on a SAB plate. After incubation, the mould grew from it. (B) Colonial appearance of flat, radially folded, waxy, yellow-cream colonies on SAB. (C-E) Wide hyphae and club-shaped spores with knob-like tips demonstrated with lactophenol cotton blue stain. (F) The thin ballistoconidia in the primary culture. All scale $10 \mu \mathrm{m}$.

At this stage, the patient's antifungal therapy was modified. Liposomal amphotericin was continued, but caspofungin was replaced by voriconazole (loading dose of $6 \mathrm{mg} / \mathrm{kg}$ BID for 1 day, then $4 \mathrm{mg} / \mathrm{kg}$ BID). In the absence of consensus guidelines or recommenda- 
tions on the management of basidiobolomycosis, and the lack of antifungal susceptibility results for this particular isolate at this stage, this antifungal combination was chosen based on several case reports describing successful use of azoles and/or amphotericin in treating such infections. The fungal isolate was then sent to the Centre of Expertise in Mycology of Radboud University Medical Centre/Canisius Wilhelmina Hospital, Nijmegen, The Netherlands for susceptibility testing. The isolate was identified by ITS and LSU sequencing as being a novel species within the genus Basidiobolus. Antifungal susceptibility testing was performed according to CLSI protocols and yielded the following MIC values: amphotericin $1 \mu \mathrm{g} / \mathrm{mL}$; voriconazole and itraconazole $>16 \mu \mathrm{g} / \mathrm{mL}$; fluconazole $>64 \mu \mathrm{g} / \mathrm{mL}$; anidulafungin and micafungin $>16 \mu \mathrm{g} / \mathrm{mL}$. As the disease progressed, the same mould also grew from multiple urine samples. Histopathology examination of the thrombus and of a section of the small intestine showed fungal elements with features compatible with zygomycetes (Figure 3).

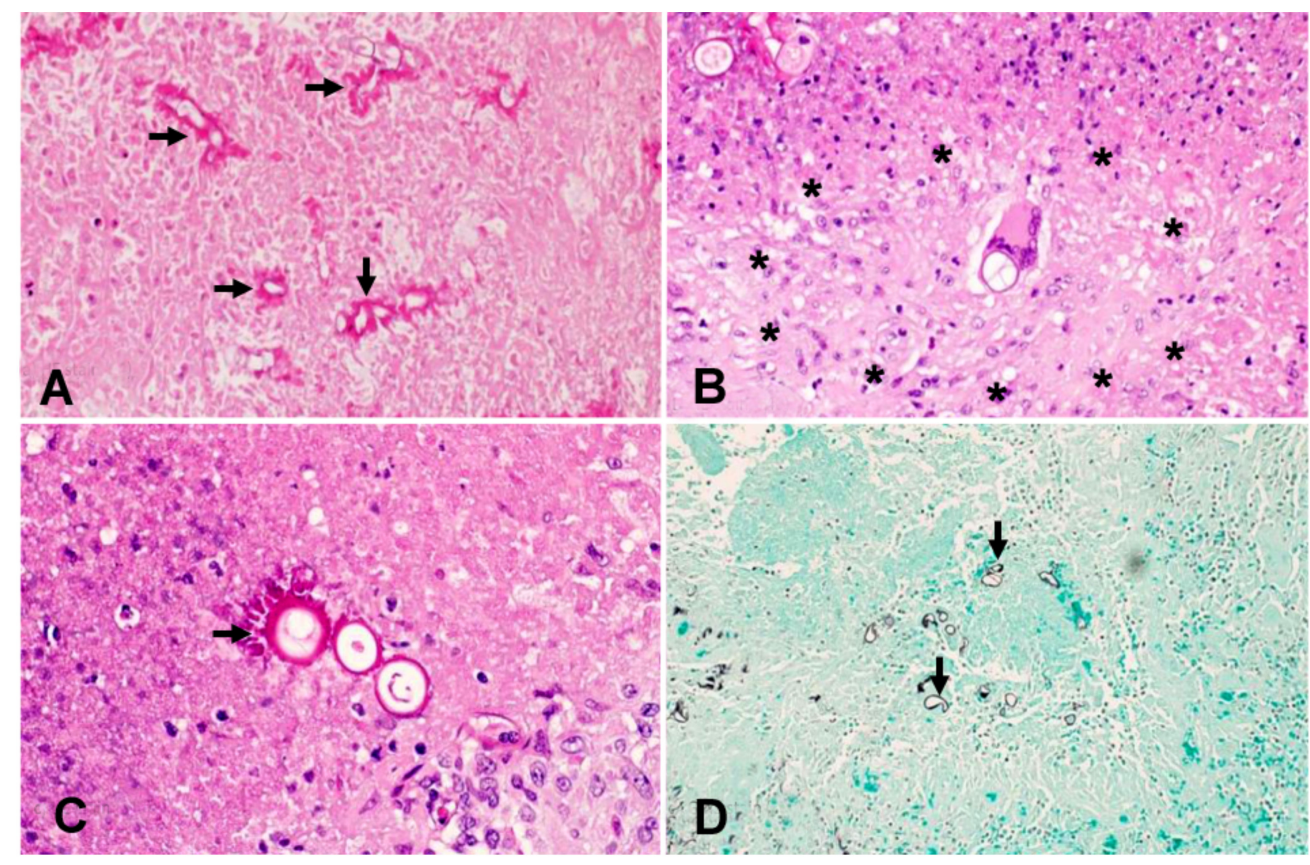

Figure 3. Histopathology findings of a section of small intestine: (A) HE stain $(4 \times)$, within the necrotic areas, there are numerous fungal elements (arrows) invading all the layers beyond the mucosa. The fungi are characterised by thin walls and broad septate hyphae in keeping with basidiobolomycosis. (B) HE stain $(40 \times)$, the fungal hyphae are associated with dense fibro-inflammatory reaction with numerous necrotising granulomas. In this image, a granuloma's border is marked by asterisks. (C) HE stain $(40 \times)$, hyphae are frequently surrounded by eosinophilic material known as SplendoreHoeppli phenomenon (arrow). (D) GMS stain $(20 \times)$, the fungi appear black with GMS stain (arrows). HE: haematoxylin and eosin; GMS: Gomori methenamine silver.

Despite the aforementioned surgical interventions and optimisation of antifungal therapy, including the empiric addition of isavuconazole (with continuation of high-dose liposomal amphotericin), the disease continued to show fulminant progression, resulting in extensive angioinvasion with involvement of the right common iliac artery, the left infrapopliteal artery and the right renal artery (Figure 4), finally resulting in bilateral lower limb ischemia and infarction of the right kidney (Figure 5). 


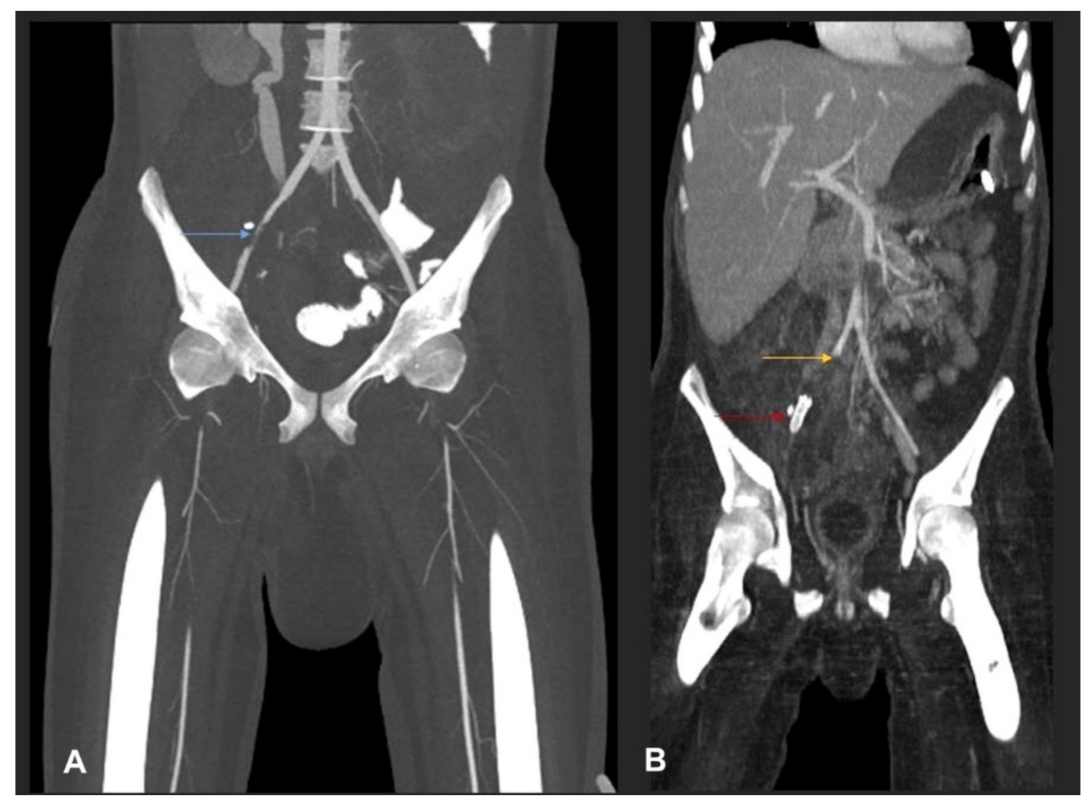

Figure 4. (A) MIP coronal CT angiography of aorta and lower limb shows a segmental narrowing and luminal irregularities of right external iliac artery (blue arrow). No occlusion. Distal run off was satisfactory. Rest of the lower limb vessels were patent and normal (not shown). (B) MIP coronal image of the aorta and lower limbs shows cut-off of the proximal right common iliac artery suggestive of thrombotic occlusion (yellow arrow). A stent is seen in the right external iliac artery (red arrow). Right iliac fossa shows extensive inflammatory phlegmonous mass-like soft tissue thickening.

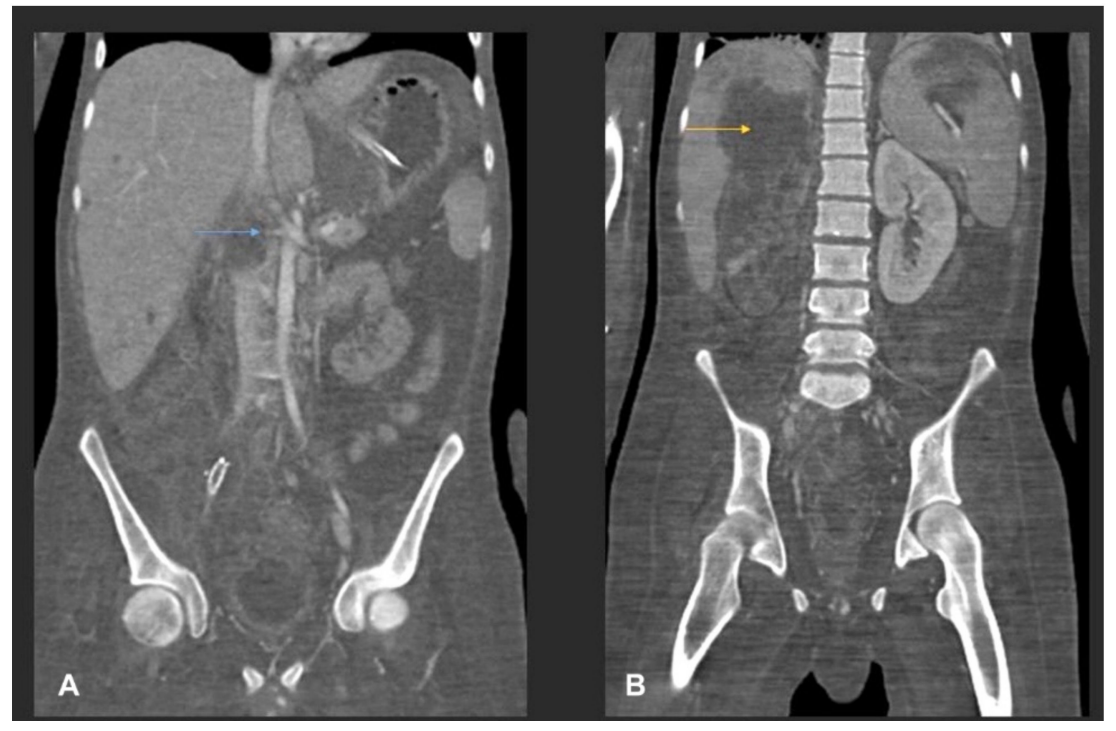

Figure 5. (A) MIP coronal images of abdomen shows a cut-off of the right proximal renal artery $1 \mathrm{~cm}$ from the ostium with adjacent inflammatory collection (blue arrow). (B) Coronal post-contrast CT shows infarction of the right kidney. A large liver abscess (yellow arrow) in the right liver lobe with extracapsular extension into the upper pole of the right kidney is also noted.

Furthermore, the hospital course was complicated by severe sepsis secondary to extensively drug-resistant Klebsiella pneumoniae bacteraemia, ventilator-associated pneumonia and deep surgical site infection, culminating in his death.

\subsection{Phylogenetic Analysis}

The analysed sequences of CBS 146281 and CBS 146282 were found to be identical. BLAST searches of ITS sequences in GenBank matched with those of Basidiobolus ranarum 
(MK501321.1 of strain PHF-MC207) with 95.96\% similarity, while LSU sequences matched with Basidiobolus sp. (MH256651.1, strain F43-5) with 100\% similarity. For further understanding of relations between species, phylogenetic analyses were conducted using ITS and LSU sequences, which included available type and reference sequences of Basidiobolus spp. known from culture (Table 1). Each dataset was analysed and compared separately prior to multilocus analysis. The final concatenated alignment comprised sequences from 17 strains, including the outgroup (Conidiobolus sp. ARSEF 7942), and consisted of 1551 nucleotide positions (ITS $=566$, LSU $=985)$, of which 1140 were conserved $($ ITS $=369$, LSU $=771), 402$ were variable $($ ITS $=197$, LSU $=205)$ and 206 were phylogenetically informative (ITS = 97, LSU = 109) (Figure 6). Phylogenetic analysis of individual and combined loci resolved eight well-supported clades, six of which corresponded to known Basidiobolus species (i.e., B. haptosporus, B. heterosporus, B. magnus, B. meristosporus, B. microsporus and B. ranarum). Strains CBS 146281 and CBS 146282 clustered in a monophyletic clade, supported by high BS and PP values (1 and 99\%, respectively), and are here described as a novel species, Basidiobolus omanensis. Additionally, the ex-type strain of B. haptosporus var. minor (CBS 310.66) clustered consistently in a well-supported linage, unrelated to the B. haptosporus core clade, and it is therefore elevated to species level below.

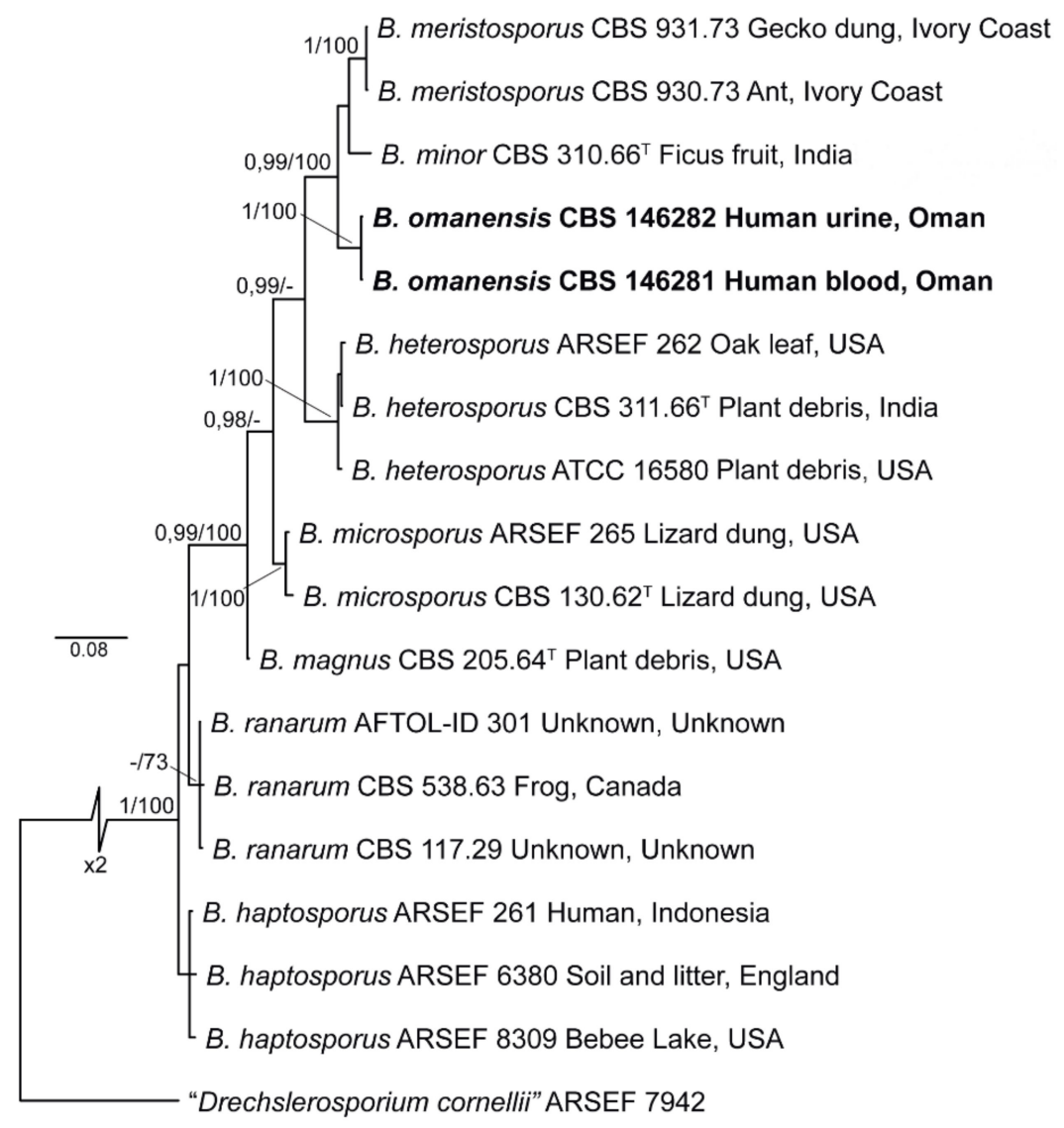

Figure 6. Maximum Likelihood (RAxML) phylogram obtained from the combined analysis of ITS and LSU sequences of Basidiobolus spp. Numbers on the nodes are Bayesian posterior probability values $(\mathrm{BI}-\mathrm{PP}) \geq 0.95$, followed by ML bootstrap values $(\mathrm{BS}) \geq 70 \%$. Novel taxa proposed in this study are indicated in bold. Ex-type strains are indicated with ${ }^{\mathrm{T}}$. The tree was rooted to Conidiobolus sp. (ARSEF 7942).

\subsection{Antifungal Susceptibility}

Antifungal susceptibility testing of B. omanensis was performed using broth microdilution protocols according to CLSI M38A2, resulting in the following MICs: amphotericin B $1 \mu \mathrm{g} / \mathrm{mL}$; fluconazole $>64 \mu \mathrm{g} / \mathrm{mL}$, itraconazole and voriconazole $>16 \mu \mathrm{g} / \mathrm{mL}$; and anidulafungin and micafungin $>16 \mu \mathrm{g} / \mathrm{mL}$. 


\subsection{Taxonomy and Description}

Basidiobolus omanensis Al-Hatmi, Sand.-Den., Balkhair, Al Adawi \& de Hoog, sp. nov.-Figure 6. MycoBank MB 839872.

Etymology: 'omanensis' refers to the country in which the fungus was first isolated.

Holotype: Oman, Muscat, from thrombus in common femoral artery of human patient, CBS 146281, designated here, preserved in metabolically inactive condition in liquid nitrogen. Living strain derived from type CBS 146281.

Description: based on colonies of CBS 146281 grown for $5 \mathrm{~d}$ at $25^{\circ} \mathrm{C}$ on MEA and SGA. On MEA, colonies grow rapidly, with an average daily growth rate of 5-6 mm; whitish, flat, waxy, glabrous and radially folded; margin entire, straight (Figure 7A,B). On SGA, colonies flat, membranous, with a smooth, glabrous and waxy appearance, becoming powdery in a later stage, with colourless aerial mycelia (Figure 7B). Radiate folds developed from the centre on both media (Figure 7A,B).

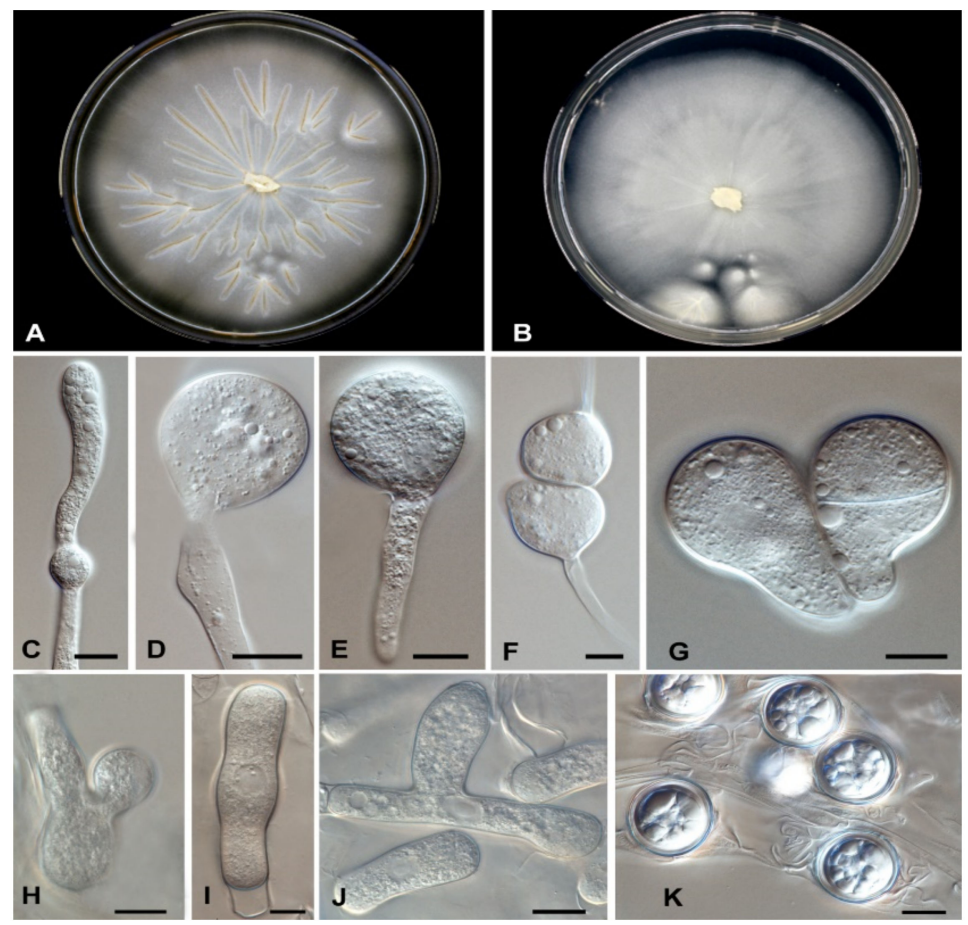

Figure 7. Basidiobolus omanensis. A colony on (A) MEA, (B) SAB after 3-5 days at $25{ }^{\circ} \mathrm{C},(\mathbf{C})$ mycelia with young chlamydospore, (D) primary conidiophore arising from mycelia, (E) primary conidia, (F) formation of zygospores, (G) mature septate conidia, (H) mature zygospores, (I,J) branched and unbranched hyphae, $(\mathbf{K})$ mature zygospores. Bars $10 \mu \mathrm{m}$.

Hyphal elements hyaline, unbranched, coenocytic or with occasional septa, broad, $5-10 \mu \mathrm{m}$ in diameter (2 to 4) (Figure 7I,J). Primary conidiophores arising from hyphal segments, unbranched, producing a single globose conidium, 4-9 $\mu \mathrm{m}$ diameter (Figure 7D,E). Secondary conidia discharged, colourless, globose to subglobose, 15-20 $\mu \mathrm{m}$ in diameter, with papilla and with or without septum, 3-5 × 1-4 $\mu \mathrm{m}$ (Figure 7G,H). Immature zygospores developed after the encounter of two hyphal segments, producing a swelling in the contact area and later becoming globose (Figure 7F). Smooth, thick-walled, spherical chlamydospores are present (Figure 7F). Numerous smooth, globose to subglobose, multinucleate zygospores, $2-4$ um in diameter present. Some of the mature zygospores contained a globule at the centre, with some space between the internal globule and the cell wall (Figure 7K).

Basidiobolus minor (Sriniv. \& Thirum.) Al-Hatmi, Sand.-Den. \& de Hoog, comb. et stat. nov.-MycoBank MB 839873. 
Basionym: Basidiobolus haptosporus var. minor Sriniv. \& Thirum., Mycopath. Mycol. Appl. 33: 60. 1967.

\section{Discussion}

We report a newly described Basidiobolus species causing refractory angioinvasive gastrointestinal disease (GIB) in a 20-year-old Omani patient with type 1 diabetes mellitus. His disease seemed to have started in the caecum with extension to the major vessels of the lower limbs. Physical examination of the lower limbs demonstrated signs of acute ischemia, confirmed by imaging studies including CT (Figures 1, 4 and 5) and by histopathology (Figure 3). From a clinical perspective, the present case is similar in main traits to other reported cases of GIB. The infection is a rare but possibly emerging disease entity, affecting immunocompetent individuals including children, and mainly occurs in hot climates worldwide [35]. In many earlier reported cases, surgery was performed without preceding diagnosis of GIB [36].

The first case of basidiobolomycosis was a subcutaneous infection reported by Joe et al. [1] from Indonesia in 1956. Gastrointestinal basidiobolomycosis has been reported worldwide, mainly in tropical and subtropical climate zones of Asia and North America [37]. Mohammadi et al. [38] reviewed more than 102 cases of GIB published between 1997 and 2018. Many cases had been reported from Saudi Arabia (62), Iran (24) and the U.S.A. (21), and to a lesser extent from Iraq (6), Kuwait and France (two cases each), Thailand, Qatar, Oman, The Netherlands and Brazil (one case each). The disease seems to be relatively common in the Middle East. Basidiobolus species cause infections in both adult and paediatric populations, while most paediatric cases were reported from Saudi Arabia [39]. Subcutaneous basidiobolomycosis has highly diverse and non-specific symptoms. On the other hand, patients with GIB may present with fever, abdominal pain, chills, weight loss, diarrhoea or constipation, and an abdominal mass can be observed [38]. As Basidiobolus is an environmental saprobe residing in soil and decaying vegetables and fruits, the route of transmission in subcutaneous disease appears to be minor trauma resulting, e.g., from an insect bite, while GIB can be acquired via intravenous catheters, intramuscular injection or ingestion of soil or faeces via contaminated food [20]. The latter transmission route may be very common, as Basidiobolus has been detected as a part of the normal mycobiome inhabiting the human gastrointestinal tract [40].

Laboratory results of our patient show an elevated white blood cells count (WBCs) of 16,700 cells $/ \mu \mathrm{L}$, with an elevated eosinophil count of 1000 cells $/ \mu \mathrm{L}$, normal glycosylated haemoglobin and deranged liver function tests. These results agreed with previously reported cases where elevated WBCs and eosinophilia were observed [37]. Abdominal imaging studies such as computed tomography (CT) are often used during the evaluation of patients with GIB [37]. In a review of abdominal imaging findings in GIB, Flicek et al. [41] commonly found abdominal masses in colon, liver or multiple sites, and bowel wall thickening. Although such findings are not diagnostic of GIB, the authors concluded that in the right clinical and epidemiological context, one should suspect GIB when an abdominal mass is seen upon CT scan [41]. In our patient, abdominal CT scans revealed a complex caecal mass adherent to the pelvic floor and to the terminal ileum with multiple hepatic lesions (Figure 1). Histological criteria such as a granulomatous reaction and mycological evidence of fungal structures are taken as suggestive of GIB [42,43]. Detailed examination of the thrombus and a section of the small intestine of our patient showed thin-walled, broad and septate hyphae, and these features are compatible with zygomycete-like fungi (Figure 3). Diagnosis of Basidiobolus infection down to the species level is typically accomplished by microbiological culture as gold standard [44]. From the thrombus specimen that was extracted from the common femoral artery, and from a urine sample, a mould was grown reminiscent of a Basidiobolus species. The colonies on PDA were expanding, subhyaline, waxy and without aerial mycelium. The wet mount preparation showed morphological characteristics consistent with Basidiobolus by production of zygospores with smooth walls, and retained short, paired protuberances leading to a 
structure known as "beaked zygospore". In addition, apical, globose primary conidia were produced with forcible conidium discharge from the conidiophores, and with pyriform secondary conidia (Figures 2 and 7). The thin ballistoconidia were seen only in the primary culture (Figure 2F). Overall, distinct morphological traits are minimal or absent between Basidiobolus species. Most of the Basidiobolus species had been distinguished on the basis of phenotypic differences such as the form of zygospores, formation of aerial hyphae, production of exogenous microspores and odour production during growth, as well as growth temperature preferences [18].

BLAST searches of the rDNA sequences of CBS 146281 and CBS 146282 revealed that the ITS sequences matched a strain of Basidiobolus ranarum with $95.96 \%$ similarity, while LSU sequences matched $100 \%$ with an unnamed Basidiobolus species. The large ITS distance to a described species, and judging from the phylogenetic ribosomal tree, led to the conclusion that our isolate is taxonomically separate from the recognised species of Basidiobolus (Figure 6) and that no formal name is available for this taxon. The new species B. omanensis is morphologically similar to other Basidiobolus species occurring in humans. The genus currently includes seven species, namely B. haptosporus, B. heterosporus, B. magnus, B. meristosporus, B. microsporus, B. minor and B. ranarum. In this study, B. omanensis is added as a novel species. Previously, only B. ranarum was reported to cause human infection, due to the fact that in most published cases, $B$. haptosporus and $B$. heterosporus have been regarded as synonyms of $B$. ranarum [45]. A previous study showed that a set of Basidiobolus spp. from Saudi Arabia clustered as a novel monophyletic lineage [7]. According to the same authors, these strains shared $99.97 \%$ ITS similarity with B. haptosporus and $99.97 \%$ with B. haptosporus var. minor, and lower similarity with B. ranarum $(99.93 \%)$, a species which is commonly linked to GIB [7]. The latter results suggest the discovery of a new and serious causal agent of GIB. Thus, our results strongly support the recognition of B. omanensis isolates as a novel species within the genus Basidiobolus; the differences observed for all phylogenetic markers are considered sufficient to propose B. omanensis as a new species of Basidiobolus. The phenotypic characteristics proposed in the literature provide insufficient resolution to differentiate the seven known species of Basidiobolus; these characters mostly include odour production along with physiological features [46-49]. However, these variations have not been systematically evaluated in larger numbers of strains. Sequencebased diagnostics are therefore required. Morphologically, the new species B. omanensis is similar to remaining species in terms of colonies with satellites, conidia, conidiophores, zygospores and chlamydospores.

Author Contributions: Each one of the authors has contributed to the production of this manuscript: Conceptualisation: A.B., A.M.S.A.-H., B.A.A., G.S.d.H. and M.S.-D.; Methodology: A.B., B.A.A., S.A.-H., A.M.S.A.-H., I.A.-B. and M.A.S.; Software; A.M.S.A.-H. and M.A.S.; Validation: B.A.A., A.M.S.A.-H. and M.S.-D.; Formal Analysis, J.F.M., A.M.S.A.-H., B.A.A., H.B.T., A.H.A.S., S.R., M.A.S., T.A.S. and Z.A.M.; Clinical Investigation: B.A.A., I.A.-B., H.B.T., A.H.A.S., S.R., M.A.S. and T.A.S., Laboratory Investigations: B.A.A., Z.A.M., T.A.S., A.M.S.A.-H., M.S.-D. and J.F.M.; Resources: A.A.R., A.A.-H., J.F.M., M.S.-D., G.S.d.H. and B.A.A.; Writing; A.B., B.A.A., A.M.S.A.-H. and M.A.S.; Review and Editing: M.S.-D., J.F.M., A.B., B.A.A., A.A.-R., A.A.-H., G.S.d.H. and A.M.S.A.-H. All authors have read and agreed to the published version of the manuscript.

Funding: This research received no external funding.

Institutional Review Board Statement: Not applicable.

Informed Consent Statement: Not applicable.

Data Availability Statement: All sequences generated in this study were submitted to GenBank.

Acknowledgments: The authors acknowledge the ICU doctors and vascular surgeons at Sultan Qaboos University Hospital for the special care given to this patient. We are indebted to Athar Al-Khirbash, pharmacist, for her efforts to obtain isavuconazole outside the hospital's formulary. Elizabeth Johnson, Mycology Reference Laboratory, Bristol, UK, is thanked for her expert advice on 
the investigation and management of the patient. We are also thankful to Khadija Al-Housni and Maather Al-Hakmani from the NMSR centre at Nizwa University for their technical support.

Conflicts of Interest: The authors declare no conflict of interest.

\section{References}

1. Joe, L.K.; Njo-Injo, T.E.; Pohan, A.; Van Der Meulen, H. Basidiobolus ranarum as a cause of subcutaneous phycomycosis in Indonesia. Arch. Dermatol. Syphol. 1956, 74, 378-383. [CrossRef]

2. Van Overeem, C. Uber ein merkwurdiges Vorkommen von Basidiobolus ranarum Eidam. Bull. Jardin Bot. 1925, 7, $423-431$.

3. Greene, C.E.; Brockus, C.W.; Currin, M.P.; Jones, C.J. Infection with Basidiobolus ranarum in two dogs. J. Am. Vet. Med. Assoc. 2002, 221, 528-532. [CrossRef]

4. Al-Maani, A.S.; Paul, G.; Jardani, A.; Nayar, M.; Al-Lawati, F.; Al-Baluishi, S.; Hussain, I.B. Gastrointestinal basidiobolomycosis: First case report from Oman and literature review. Sultan Qaboos Univ. Med. J. 2014, 14, e241.

5. Khan, Z.U.; Khoursheed, M.; Makar, R.; Al-Waheeb, S.; Al-Bader, I.; Al-Muzaini, A.; Chandy, R.; Mustafa, A.S. Basidiobolus ranarum as an etiologic agent of gastrointestinal zygomycosis. J. Clin. Microbiol. 2001, 39, 2360-2363. [CrossRef]

6. AL-Naemi, A.Q.; Khan, L.A.; AL-Naemi, I.; Amin, K.; Athlawy, Y.A.; Awad, A.; Sun, Z. A Case report of gastrointestinal basidiobolomycosis treated with voriconazole. Medicine 2015, 94, e1430. [CrossRef]

7. Al Bshabshe, A.; Joseph, M.R.P.; Al Hakami, A.M.; Al Azraqi, T.; Al Humayed, S.; Hamid, M.E. Basidiobolus haptosporus-like fungus as a causal agent of gastrointestinal basidiobolomycosis. Med. Mycol. 2020, 58, 264-267. [CrossRef] [PubMed]

8. Schüßler, A.; Schwarzott, D.; Walker, C. A new fungal phylum, the Glomeromycota: Phylogeny and evolution. Mycol. Res. 2001, 105, 1413-1421. [CrossRef]

9. Hibbett, D.S.; Binder, M.; Bischoff, J.F.; Blackwell, M.; Cannon, P.F.; Eriksson, O.E.; Huhndorf, S.; James, T.; Kirk, P.M.; Lücking, R.; et al. A higher-level phylogenetic classification of the Fungi. Mycol. Res. 2007, 111, 509-547. [CrossRef]

10. Kwon-Chung, K.J. Taxonomy of fungi causing mucormycosis and entomophthoramycosis (zygomycosis) and nomenclature of the disease: Molecular mycologic perspectives. Clin. Infect. Dis. 2012, 54 (Suppl. S1), S8-S15. [CrossRef] [PubMed]

11. Humber, R.A. Entomophthoromycota: A new phylum and reclassification for entomophthoroid fungi. Mycotaxon 2012, 120, 477-492. [CrossRef]

12. Gryganskyi, A.P.; Humber, R.A.; Miadlikovska, J.; Smith, M.E.; Wu, S.; Voigt, K.; Walther, G.; Anishchenko, I.M.; Vilgalys, R. Molecular phylogeny of the Entomophthoromycota. Mol. Phylogenet. Evol. 2012, 65, 682-694. [CrossRef]

13. Gryganskyi, A.P.; Humber, R.A.; Smith, M.E.; Hodge, K.; Huang, B.; Voigt, K.; Vilgalys, R. Phylogenetic lineages in Entomophthoromycota. Persoonia 2013, 30, 94-105. [CrossRef]

14. Spatafora, J.W.; Chang, Y.; Benny, G.L.; Lazarus, K.; Smith, M.E.; Berbee, M.L.; Bonito, G.; Corradi, N.; Grigoriev, I.; Gryganskyi, A.; et al. A phylum-level phylogenetic classification of zygomycete fungi based on genome-scale data. Mycologia 2016, 108, 1028-1046. [CrossRef] [PubMed]

15. Vilela, R.; Mendoza, L. Human pathogenic Entomophthorales. Clin. Microbiol. Rev. 2018, 31, e00014-18. [CrossRef] [PubMed]

16. Eidam, E. Basidiobolus eine neue gattung der Entomophthoracean. Beitr. Biol. Pflanz. 1886, 4, 181-251.

17. Claussen, M.; Schmidt, S. Differentiation of Basidiobolus spp. isolates: RFLP of a diagnostic PCR amplicon matches sequence-based classification and growth temperature preferences. J. Fungi 2021, 7, 110. [CrossRef] [PubMed]

18. El-Shabrawi, M.H.F.; Arnaout, H.; Madkour, L.; Kamal, N.M. Entomophthoromycosis: A challenging emerging disease. Mycoses 2014, 57, 132-137. [CrossRef]

19. Chetambath, R.; Deepa Sarma, M.S.; Suraj, K.P.; Jyothi, E.; Mohammed, S.; Philomina, B.J.; Ramadevi, S. Basidiobolus: An unusual cause of lung abscess. Lung India 2010, 27, 89-92. [CrossRef]

20. Shaikh, N.; Hussain, K.A.; Petraitiene, R.; Schuetz, A.N.; Walsh, T.J. Entomophthoramycosis: A neglected tropical mycosis. Clin. Microbiol. Infect. 2016, 22, 688-694. [CrossRef]

21. James, T.Y.; Kauff, F.; Schoch, C.L.; Matheny, P.B.; Hofstetter, V.; Cox, C.J.; Celio, G.; Gueidan, C.; Fraker, E.; Miadlikowska, J.; et al. Reconstructing the early evolution of fungi using a six-gene phylogeny. Nature 2006, 443, 818-822. [CrossRef] [PubMed]

22. Moller, E.M.; Bahnweg, G.; Sandermann, H.; Geiger, H.H. A simple and efficient protocol for isolation of high molecular weight DNA from filamentous fungi, fruit bodies, and infected plant tissues. Nucleic Acids Res. 1992, 20, 6115-6116. [CrossRef]

23. Vilgalys, R.; Hester, M. Rapid genetic identification and mapping of enzymatically amplified ribosomal DNA from several Cryptococcus species. J. Bacteriol. 1990, 172, 4238-4246. [CrossRef] [PubMed]

24. Gargas, A.; Taylor, J.W. Polymerase chain reaction (PCR) primers for amplifying and sequencing $18 \mathrm{~S}$ rDNA from lichenized fungi. Mycologia 1992, 84, 589-592. [CrossRef]

25. White, T.J.; Bruns, T.; Lee, S.; Taylor, J. Amplification and direct sequencing of fungal ribosomal RNAgenes for phylogenetics. In PCR Protocols: A Guide to Methods and Applications; Innis, M.A., Gelfand, D.H., Sninsky, J.J., White, T.J., Eds.; Academic Press: Cambridge, MA, USA, 1990; pp. 315-322.

26. Katoh, K.; Standley, D.M. MAFFT multiple sequence alignment software v. 7: Improvements in performance and usability. Mol. Biol. Evol. 2013, 30, 772-780. [CrossRef] [PubMed]

27. Miller, M.A.; Pfeier, W.; Schwartz, T. The Cipres Science Gateway: Enabling high-impact science for phylogenetics researchers with limited resources. In Proceedings of the 1st Conference of the Extreme Science and Engineering Discovery Environment: Bridging from the Extreme to the Campus and Beyond, Chicago, IL, USA, 16-20 July 2012; pp. 1-8. 
28. Stamatakis, A. RAxML version 8: A tool for phylogenetic analysis and post-analysis of large phylogenies. Bioinformatics 2014, 30, 1312-1313. [CrossRef]

29. Huelsenbeck, J.P.; Ronquist, F. MrBayes: Bayesian inference of phylogeny. Bioinformatics 2001, 17, 754-755. [CrossRef] [PubMed]

30. Ronquist, F.; Huelsenbeck, J.P. MrBayes 3: Bayesian phylogenetic inference under mixed models. Bioinformatics 2003, 19, 1572-1574. [CrossRef]

31. Nylander, J.A.A. MrModeltest v2. Program Distributed by the Author; Evolutionary Biology Centre, Uppsala University: Uppsala, Sweden, 2004.

32. Mason-Gamer, R.; Kellogg, E. Testing for phylogenetic conflict among molecular data sets in the tribe Triticeae (Gramineae). Syst. Biol. 1996, 45, 524-545. [CrossRef]

33. Wiens, J.J. Testing phylogenetic methods with tree congruence: Phylogenetic analysis of polymorphic morphological characters in phrynosomatid lizards. Syst. Biol. 1998, 47, 427-444. [CrossRef]

34. CLSI. Reference Method for Broth Dilution Antifungal Susceptibility Testing of Filamentous Fungi, 3rd ed.; Clinical and Laboratory Standards Institute: Wayne, PA, USA, 2017.

35. Rabie, M.E.; Al Qahtani, A.S.; Jamil, S.; Mikhail, N.T.; El Hakeem, I.; Hummadi, A.; Elshaar, K.E.; Abdelraheem, I.; Saudi, D. Gastrointestinal basidiobolomycosis: An emerging potentially lethal fungal infection. Saudi. Surg. J. 2019, 7, 1-9.

36. Geramizadeh, B.; Heidari, M.; Shekarkhar, G. Gastrointestinal basidiobolomycosis, a rare and under-diagnosed fungal infection in immunocompetent hosts: A review article. Iran. J. Med. Sci. 2015, 40, 90-97. [PubMed]

37. Vikram, H.R.; Smilack, J.D.; Leighton, J.A.; Crowell, M.D.; De Petris, G. Emergence of gastrointestinal basidiobolomycosis in the United States, with a review of worldwide cases. Clin. Infect. Dis. 2012, 54, 1685-1691. [CrossRef]

38. Mohammadi, R.; Ansari Chaharsoghi, M.; Khorvash, F.; Kaleidari, B.; Sanei, M.H.; Ahangarkani, F.; Abtahian, Z.; Meis, J.F.; Badali, H. An unusual case of gastrointestinal basidiobolomycosis mimicking colon cancer; Literature and review. J. Mycol. Med. 2019, 29, 75-79. [CrossRef]

39. El-Shabrawi, M.H.; Kamal, N.M.; Jouini, R.; Al-Harbi, A.; Voigt, K.; Al-Malki, T. Gastrointestinal basidiobolomycosis: An emerging fungal infection causing bowel perforation in a child. J. Med. Microbiol. 2011, 60, 1395-1402. [CrossRef] [PubMed]

40. Gouba, N.; Drancourt, M. Digestive tract mycobiota: A source of infection. Med. Mal. Infect. 2015, 45, 9-16. [CrossRef]

41. Flicek, K.T.; Vikram, H.R.; De Petris, G.D.; Johnson, C.D. Abdominal imaging findings in gastrointestinal basidiobolomycosis Abdom. Imaging 2015, 40, 246-250. [CrossRef]

42. Al Jarie, A.; Al Azraki, T.; Al Mohsen, I.; Al Jumaah, S.; Almutawa, A.; Mohd Fahim, Y.; Al Shehri, M.; Abu Dayah, A.; Ibrahim, A.; Maw Shabana, M. Basidiobolomycosis: Case series. J. Mycol. Med. 2011, 21, 37-45. [CrossRef]

43. Al-Masqari, M.; Al-Maani, A.; Ramadhan, F. Gastrointestinal Basidiobolomycosis: An emerging fungal infection of the gastrointestinal tract, the Royal Hospital (Sultanate of Oman) experience. Pediatr. Inf. Dis. 2021, 3, 46-49.

44. Balkhair, A.; Al Wahaibi, A.; Al-Qadhi, H.; Al-Harthy, A.; Lakhtakia, R.; Rasool, W.; Ibrahim, S. Gastrointestinal basidiobolomycosis: Beware of the great masquerade a case report. IDCases 2019, 18, e00614. [CrossRef]

45. De Hoog, G.S.; Guarro, J.; Gené, J.; Ahmed, S.; Al-Hatmi, A.M.S.; Figueras, M.J.; Vitale, R.G. Atlas of Clinical Fungi, 4th ed.; Foundation Atlas of Clinical Fungi: Hilversum, The Netherlands, 2020.

46. Drechsler, C. A southern Basidiobolus forming many sporangia from globose and from elongated adhesive conidia. J. Wash. Acad. Sci. 1955, 45, 49-56.

47. Benjamin, R.K. A new Basidiobolus that forms microspores. Aliso 1962, 5, 223-233. [CrossRef]

48. Drechsler, C. An odorous Basidiobolus often producing conidia plurally and forming some diclinous sexual apparatus. Am. J. Bot. 1964, 51, 770-777. [CrossRef]

49. Hutchison, J.A.; King, D.S.; Nickerson, M.A. Studies on temperature requirements, odor production and zygospore wall undulation of the genus Basidiobolus. Mycologia 1972, 64, 467-474. [CrossRef] [PubMed] 